Petr Kozel - Lucie Orlikova - Sarka Michalcova*

\title{
THE MODIFIED RURAL POSTMAN PROBLEM IN VEHICLE ROUTE OPTIMIZATION
}

The submitted paper deals with designing routes of the vehicles, which provide the transport network services. We limit our focus to such tasks, where the priority is the edge service in the transport network and the initial problem is finding an Eulerian path. Regarding to real-life problems, this contribution presents such procedure of solving, which takes into account both the existence of a mixed transport network containing one-way roads and the existence of a wider transport network. In this network, there are only selected edges with possibility of the effective passages. This problem can be solved by the modified Rural Postman Problem assuming the strongly connected network. Linear programming is a suitable tool for designing optimal routes of service vehicles.

Keywords: Linear programming, Eulerian path, vehicle routing tasks, the Rural Postman Problem, municipal waste collection

\section{Introduction}

Vehicle route planning is an inherent part of the decision making process of the all subjects providing a transport network service (such as cleaning and maintenance of roads, municipal waste collection, separated municipal waste collection etc.). Generally, the underlying problem is to design service vehicles routes while taking into account the chosen optimization criterion and respecting all of the substantial constraints resulting from the real traffic, too. Particularly, the most commonly used optimization criterion for designing service vehicle routes is minimizing the total distance travelled. In general, tasks relating to the route planning and optimization can be divided into two basic groups. The first one includes such problems that solve a point in a transport network - a vertex (customer, depot etc.) [1], [2] and the aim of these tasks is to find the minimum Hamiltonian cycle. The next group represents such tasks, in which the subject of service is a road - an edge (snow cleaning, street cleaning etc.) and the basic objective is to determine an Eulerian path. In this paper, we limit our focus to problems of the second set of tasks.

\section{Basic problem formulation}

Let the real transport network be represented by graph $N(V, E, l)$ with three attributes: $V=1, \ldots, m$ as set of vertices, $E=1, \ldots, m$ denotes a set of edges and $l_{i j}$ (where $i, j \in V$ ) represents an edge evaluation and denote the road length (in kilometres). Generally, the aim is to find a route that passes through each edge at least once with the minimal length and also begins and ends at the depot. This problem was first formulated by Mei-Ko-Kuan and in the professional literature is known as the Chinese Postman Problem [3]. Assuming that all conditions for existence of Eulerian path are met the Edmonds' algorithm [4] can be used to find a solution of this problem. However, if some of these conditions fails to be met, the application of an alternative approach becomes necessary to eliminate this shortage. Principally, it is vital to identify those edges, which are used repeatedly on the route. In practice, these are unproductive passages through some sections. For this, the so called minimum matching principle [5] is commonly applied to identify the repeatedly included edges, in detail in [6]. Nevertheless, the disadvantage of this approach is the necessity to design some substitute transport network, in which the duplicate edges can be identified by using the minimum matching principle. In this respect, we consider more appropriate procedure for identifying duplicate edges by determining the number of passages through each edge of the transport network [5]. Above all, it is not necessary to create a substitute network in the framework of this approach. Information in the matrix of distances $l_{i j}$ corresponding with the default network $N$ is included in the mathematical model. In addition, this matrix contains only values corresponding to the vertices that are connected by the edge. This fact is expressed as "exists" $l_{i j}$.

\subsection{Basic problem formulation - mathematical model}

Mathematical model of the above mentioned issues can be formulated as follows:

$$
\begin{aligned}
& \operatorname{Min} \sum_{i=1}^{m} \sum_{\substack{j=1 \\
\text { exists } l_{i j}}}^{m} l_{i j} \cdot z_{i j} \\
& z_{i j}+z_{j i} \geq 1 \quad \text { for } i, j=1, \ldots, m, \text { where exists } l_{i j} \\
& \sum_{\substack{j=1 \\
\text { exist } l_{i j}}}^{m} z_{i j}=\sum_{\substack{j=1 \\
\text { exists } l_{i j}}}^{m} z_{i j} \text { for } i=1, \ldots, m \\
& z_{i j} \in Z^{+} \quad \text { for } i=1, \ldots, m, \text { where exists } l_{i j}
\end{aligned}
$$

\footnotetext{
* Petr Kozel, Lucie Orlikova, Sarka Michalcova

Department of Mathematical Methods in Economics, Faculty of Economics, Technical University of Ostrava, Czech Republic E-mail: petr.kozel@vsb.cz
} 


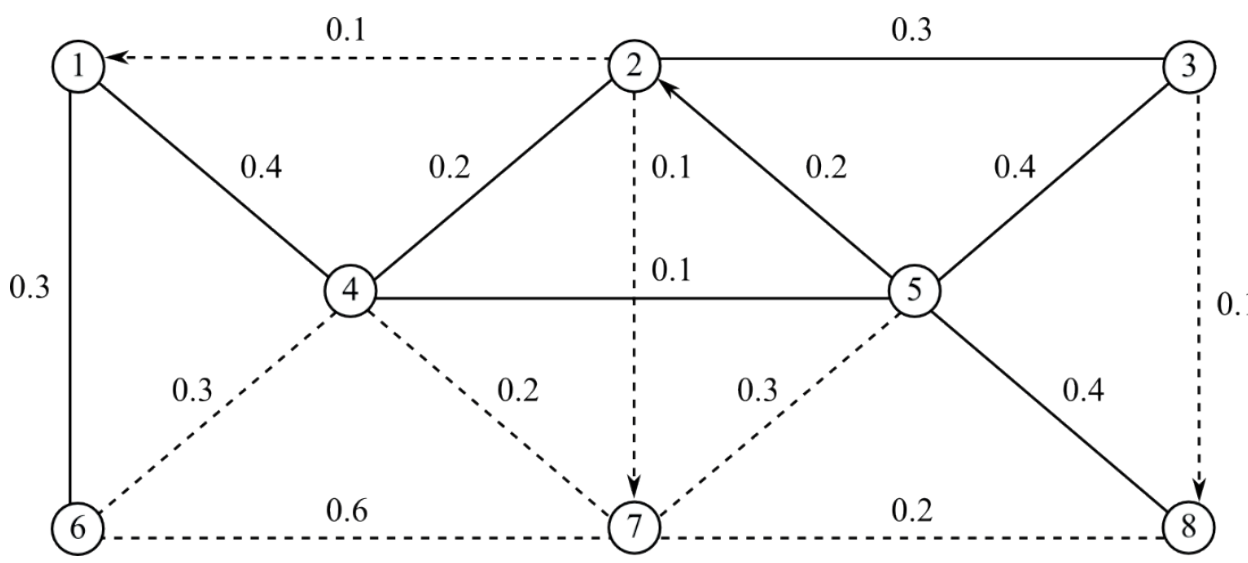

Figure 1 The network $N$ with marked subnets $N_{1}$ and $N_{2}$

The objective function (1) gives e.g. the total distance travelled during the waste municipal collection. The variable $z_{i j}$ is an integer representing the number of passages through the edge $i j$. The conditions (2) ensure that every existing edge (exists $l_{i j}$ ) is included at least once. The condition (3) ensures that each visited vertex will be subsequently abandoned. The obligatory condition (4) indicates that the variable $z_{i j}$ is a nonnegative integer. Numerical experiments with this model were published in [6].

\section{Mathematical model for real networks - modified approach}

The mathematical model (1) - (4) can be used only for undirected transport network without further specific requirements. However, with regard to the practice, it is necessary to take into account several other requirements:

- existence of a mixed transport network, which contains directed and undirected edges (e.g. one-way roads in practice);

- existence of a wider transport network, in which the service of some edges is required (e.g. this corresponds to the situation when the certain set of roads is served and other roads can be used for efficient passages).

Taking into account the above-formulated requirements of the real traffic, both the approach and the mathematical model are presented in the following text.

\subsection{Formulation of modified task formulation}

The specified network $N(V, E, l)$ defines a mixed graph. This network has three attributes and can be divided to several subnets. In our case there are two subnets. The first one $N_{1}\left(V_{1}, E_{1}, d\right)$ represents a set of edges, which have to be served at least once. The second subnet $N_{2}\left(V_{2}, E_{2}, e\right)$ determines a set of edges, which can be used for efficient passages. The constant $l_{i j}$ denotes edge evaluation $i j, d_{i j} \subset l_{i j}$ and $e_{i j} \subset l_{i j}$. Hence, the mutual relation can be expressed mathematically as follows: $N=N_{1} \cup N_{2}$. The aim of this problem is to find such route, which passes through all the edges of the subgraph $N_{1}$ at least once with possibility of using the edges from the subgraph $N_{2}$ so that the total length is minimal. The request route starts and ends at the initial vertex.

The following requirement must be met at the same time: firstly, the directed edges, located both in subnet $N_{1}$ and $N_{2}$, can be passed only in the edge direction. This requirement corresponds to the one-way roads in practice.

The model case is presented in Figure 1, where the network $N$ is created by 8 vertices and 16 edges. Subnet $N_{1}$ (indicated by solid lines) represents the edges, which have to be served at least once. The subnet $N_{2}$ (indicated by dashed lines) shows the edges, which may be used for efficient passages.

\subsection{Sufficient preconditions to admissibility of the solution}

Unlike the classical version of the Rural Postman Problem [7], the strongly connected subnet $N_{1}$ is a basic assumption of the approach presented in this paper. If this subnet is not strongly connected, the resulting solution obtained by the proposed method may include subnets in the form of isolated closed trails. However, assuming the strongly connected subnet $N_{1}$, this situation cannot occur. This claim is described in detail and confirmed by the proof in the following text. There are introduced two auxiliary concepts to demonstrate this proof:

- an obligatory edge - an edge belonging to the subset $E_{1}$,

- an obligatory vertex - a vertex that incidents with an obligatory edge at least once.

Assumptions:

1. The edge evaluation is the set of positive real numbers.

2. All just required obligatory vertices are located in the subgraph $N_{1}$.

3. The subgraph $N_{1}$ is strongly connected, thus for each two vertices $i, j$ it is possible to find an oriented path from $i$ to $j$ and $j$ to $i$, too.

The statement: The resulting route obtained by the proposed method is a single closed trail including all required obligatory edges, therefore it does not include two or more isolated closed trails that do not have a common vertex.

\section{Proof by contradiction:}

1. Let the resulting route contains an isolated closed trail that does not have a common obligatory vertex with a rest of the route

2. Both isolated closed trail and the rest of the route must contain a obligatory edge with at least one positive evaluation.

3. However, all obligatory vertices belong to the subgraph $N_{1}$ that is strongly connected and therefore there is a route, which 


\begin{tabular}{ccccccccccccccccccc}
\hline \multicolumn{10}{c}{ Input data } \\
\hline$i j$ & 14 & 16 & 23 & 24 & 25 & 32 & 35 & 41 & 42 & 45 & 52 & 53 & 54 & 58 & 61 & 85 \\
$d_{i j}$ & 0.4 & 0.3 & 0.3 & 0.2 & $\mathrm{~T}$ & 0.3 & 0.4 & 0.4 & 0.4 & 0.1 & 0.2 & 0.4 & 0.1 & 0.4 & 0.3 & 0.4 \\
$i j$ & 12 & 21 & 27 & 38 & 46 & 47 & 57 & 64 & 67 & 72 & 74 & 75 & 76 & 78 & 83 & 0.3 \\
$e_{i j}$ & $\mathrm{~T}$ & 0.1 & 0.1 & 0.1 & 0.3 & 0.2 & 0.3 & 0.3 & 0.6 & $\mathrm{~T}$ & 0.2 & 0.3 & 0.6 & 0.2 & $\mathrm{~T}$ & \\
\hline
\end{tabular}

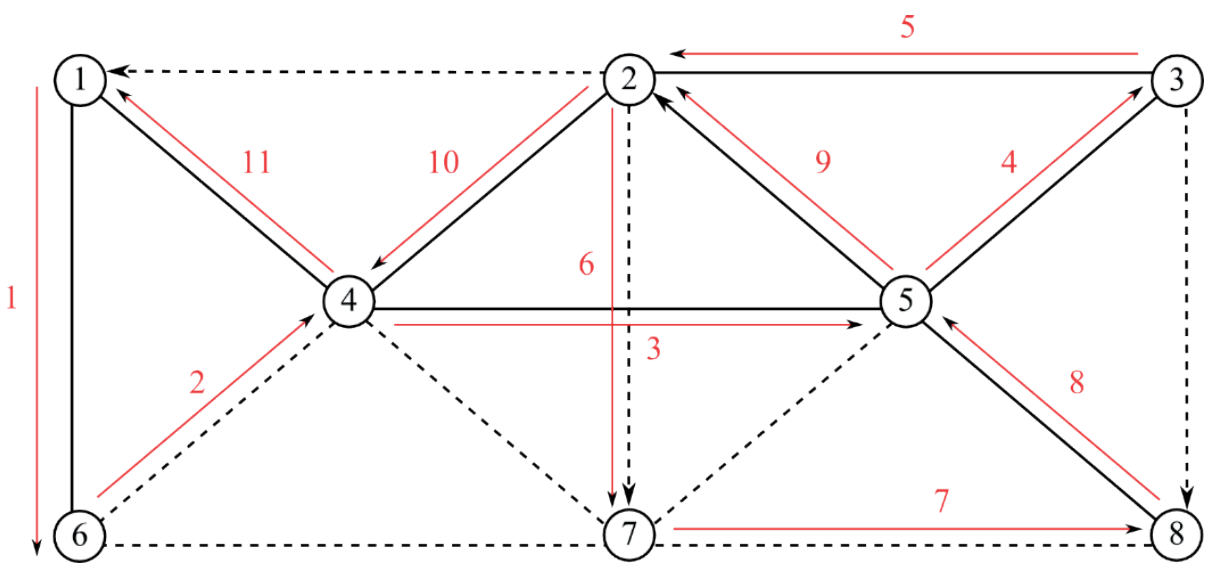

Figure 2 The use of edges of the subnets $N_{1}$ and $N_{2}$ in the optimal solution

is composed only of the obligatory edges and connects any selected obligatory vertex from the isolated closed trail with one of the final (obligatory) vertices of the obligatory edge in the rest of the route. On this route, there must necessarily be an obligatory vertex, which is the final vertex of the obligatory edge of the isolated closed trail and at the same time, it is the final vertex of the obligatory edge that belongs to the rest of the route. It is a dispute with that assumption.

\section{Mathematical model of the modified task}

To find the optimal route, it is possible to use a mathematical model that determines, how many times the edge $i j$ is used in the route. This model (5) - (9) is based on the previously mentioned model (1) - (4). Data corresponding to the subnets $N_{1}$ and $N_{2}$ enter into the mathematical model. The evaluation of existing edges in subnet $N_{1}$ is represented by a constant $d_{i j}$ and the evaluation of existing edges in subnet $N_{2}$ is represented by a constant $e_{i j}$. This method of constant determination ensures that the existing edges are used and what is more, it corresponds to the input matrices, which are sparse. The dynamic declaration is used in the computational environment.

The possibility of passages through the edge against its direction is penalized by a prohibitive constant $T$, the corresponding relationship is defined in [6]. An example of the sparse matrix with the prohibitive constant $T$ corresponding to Figure 1 is given in Table 1.

The number of the edge passages $i j$ - mathematical model [8]:

$$
\begin{aligned}
& \operatorname{Min} \sum_{i=1}^{m} \sum_{\substack{j=1 \\
\text { exists } d_{j j}}}^{m} d_{i j} \cdot z_{i j}+\sum_{i=1}^{m} \sum_{\substack{j=1 \\
\text { exists } e_{j j}}}^{m} e_{i j} \cdot y_{i j} \\
& z_{i j}+z_{j i} \geq 1 \quad \text { for } i, j=1, \ldots, m, \text { where exists } d_{i j}
\end{aligned}
$$

$$
\begin{aligned}
& \sum_{\substack{j=1 \\
\text { exists } d_{i j}}}^{m} z_{i j}+\sum_{\substack{j=1 \\
\text { exists } e_{i j}}}^{m} y_{i j}=\sum_{\substack{j=1 \\
\text { exists } d_{i j}}}^{m} z_{i j}+\sum_{\substack{j=1 \\
\text { exist } e_{i j}}}^{m} y_{i j} \text { for } i=1, \ldots, m \\
& z_{i j} \in Z_{0}^{+} \text {for } i=1, \ldots, m, \text { where exists } d_{i j} \\
& y_{i j} \in Z_{0}^{+} \text {for } i=1, \ldots, m, \text { where exists } e_{i j}
\end{aligned}
$$

The objective function (5) gives e.g. the total distance travelled during the service route. The first part of the expression is the distance traveled during the edges service of the subnet $N_{1}$, and the second part represents the distance traveled by using edges from the subnet $N_{2}$. The variable $z_{i j}$ is an integer variable that expresses the number of edge runs in the subnet $N_{1}$. The variable $y_{i j}$ is an integer variable which determines the number of passages through edges of the subnet $N_{2}$. Condition (6) ensure that each existing edge in the subnet $N_{1}$ is included in the route at least once. The condition (7) means that each visited vertex will be subsequently abandoned. The obligatory conditions (8) and condition (9) ensure that variables $z_{i j}$ and $y_{i j}$ may be only the nonnegative integer.

\section{Computational experiments}

This chapter focuses on experiments, which were realized with mathematical model (5)-(9) in computational environment Xpress-IVE [9], [10]. Prior to presenting final results, the solution procedure is given by an illustration example. Let us suppose the network as depicted in Figure 1. Firstly, the solution may be obtained assuming that it is not possible to use the other edges than the edges of the subnet $N_{1}$. The optimal route (1-4-5-8-5-2-3-52-4-1-6-1) length corresponding to this solution is $3.6 \mathrm{~km}$.

Secondly, the optimal solution can be also achieved by using edges of the subnet $N_{2}$. This optimal route (1-6-4-5-3-2-7-8-5-2-4-1) with length $2.9 \mathrm{~km}$ is shown in Figure 2. In accordance with this 
Table 2 Results of numerical experiments 1 - 6

\begin{tabular}{|c|c|c|c|c|c|c|c|}
\hline \multicolumn{4}{|c|}{ Exp. 1: (133 vert. / 168 edg. / 8 one-way) } & \multicolumn{4}{|c|}{ Exp. 4: (114 vert. / 147 edg. / 15 one-way) } \\
\hline \multirow{2}{*}{ V1 } & 104 / 3 & V & $64 / 5$ & \multirow{2}{*}{ V1 } & $99 / 8$ & \multirow{2}{*}{$\mathrm{V} 2$} & $48 / 7$ \\
\hline & $108.3[\mathrm{~km}]$ & $\sqrt{2}$ & $82.1[\mathrm{~km}], 24.2 \%$ & & $70.3[\mathrm{~km}]$ & & $67.1[\mathrm{~km}], 4.6 \%$ \\
\hline \multicolumn{4}{|c|}{ Exp. 2: (128 vert. / 166 edg. / 18 one-way) } & \multicolumn{4}{|c|}{ Exp. 5: (102 vert. / 126 edg. / 7 one-way) } \\
\hline \multirow{2}{*}{ V1 } & $130 / 10$ & V & $36 / 8$ & \multirow{2}{*}{ V1 } & $84 / 4$ & \multirow{2}{*}{$\mathrm{V} 2$} & $42 / 3$ \\
\hline & $112.3[\mathrm{~km}]$ & $\sqrt{2}$ & $95.8[\mathrm{~km}], 14.7 \%$ & & $62.1[\mathrm{~km}]$ & & $57.6[\mathrm{~km}], 7.2 \%$ \\
\hline \multicolumn{4}{|c|}{ Exp. 3: (72 vert. / 98 edg. / 12 one-way) } & \multicolumn{4}{|c|}{ Exp. 6: (104 vert. / 131 edg. / 6 one-way) } \\
\hline \multirow{2}{*}{$\mathrm{V} 1$} & $70 / 5$ & \multirow{2}{*}{$\mathrm{V} 2$} & $28 / 7$ & \multirow{2}{*}{ V1 } & $86 / 3$ & \multirow{2}{*}{$\mathrm{V} 2$} & $45 / 3$ \\
\hline & $58.6[\mathrm{~km}]$ & & $50[\mathrm{~km}], 14.7 \%$ & & $69.6[\mathrm{~km}]$ & & $66.9[\mathrm{~km}], 3.9 \%$ \\
\hline
\end{tabular}

Table 3 Results of numerical experiments $7-10$

\begin{tabular}{|c|c|c|c|c|c|c|c|}
\hline \multicolumn{4}{|c|}{ Exp. 7: (185 vert. / 239 edg. / 9 one-way) } & \multicolumn{4}{|c|}{ Exp. 9: (157 vert. / 213 edg. / 10 one-way) } \\
\hline \multirow{2}{*}{ V1 } & $139 / 7$ & \multirow{2}{*}{$\mathrm{V} 2$} & $100 / 2$ & \multirow{2}{*}{$\mathrm{V} 1$} & $126 / 7$ & \multirow{2}{*}{$\mathrm{V} 2$} & 87 / 3 \\
\hline & $112.3[\mathrm{~km}]$ & & $95.9[\mathrm{~km}], 14.6 \%$ & & $90.5[\mathrm{~km}]$ & & $77.8[\mathrm{~km}], 14 \%$ \\
\hline \multicolumn{4}{|c|}{ Exp. 8: (147 vert. / 186 edg. / 8 one-way) } & \multicolumn{4}{|c|}{ Exp. 10: (139 vert. / 186 edg. / 7 one-way) } \\
\hline \multirow{2}{*}{ V1 } & $123 / 5$ & \multirow{2}{*}{$\mathrm{V} 2$} & $63 / 3$ & \multirow{2}{*}{ V1 } & $122 / 5$ & \multirow{2}{*}{$\mathrm{V} 2$} & $64 / 2$ \\
\hline & $96.9[\mathrm{~km}]$ & & $82.9[\mathrm{~km}], 14.4 \%$ & & $90.4[\mathrm{~km}]$ & & $74.4[\mathrm{~km}], 17.7 \%$ \\
\hline
\end{tabular}

Table 4 Results of numerical experiment - 11 (real problem)

\begin{tabular}{|c|c|c|c|}
\hline \multicolumn{4}{|c|}{ Exp. 11: (3706 vert. / 6446 edg. / 1012 one-way) } \\
\hline \multirow{2}{*}{ V1 } & $2063 / 249$ & \multirow{2}{*}{$\mathrm{V} 2$} & $4383 / 763$ \\
\hline & $280[\mathrm{~km}]$ & & $268[\mathrm{~km}], 4.3 \%$ \\
\hline
\end{tabular}

solution, 3 edges of the subnet $N_{2}$ are used for the effective vehicle transits. When comparing to the original solution, the achieved saving is $0.7 \mathrm{~km}$, which is $12.1 \%$.

In the following we will present such results, which were realized using the mathematical model (5)-(9) on a set of 11 nontrivial tasks [11], [12]. For the sake of usage, the size and conditions of these tasks correspond to real problems. For the first ten tasks, all input data were generated by Wolfram Mathematica [13], [14]. Input data for last task were obtained from the real traffic of municipal waste collection, which are provided by Technical services in Olomouc. All calculations have been made for two variants (V1 and V2). To design the optimal route, the first variant enables using only edges of the subnet $N_{1}$ and the second one allows to use edges from the subnet $N_{2}$. Results with experiments 1-10 are summarized in Table 2 and Table 3. Results with the experiment 11 are summarized in Table 4.

Each of the experiments 1-11 shown in Table 2, Table 3 and Table 4 are characterised briefly by the following aspects: the number of vertices / the number of edges / the number of one-way streets and it is divided into two variants of solution V1, V2. The basic network $N_{1}$ is used in the variant V1 (the number of edges / the number of one-way streets in the network $N_{1}$ ). The second variant V2 works with the networks $N_{1}$ and $N_{2}$ (the number of edges / the number of one-way streets in $N_{2}$ ). The total route length (in $\mathrm{km}$ ) is stated in each of the variant. Furthermore, the percentage improvement of V2 compared to V1 is shown for each experiment. The computation time of all experiments was about 2 seconds.

\section{Conclusion}

This present paper deals with the planning of the optimal service vehicle route using the integer programming. We have introduced the mathematical model with constraints corresponding to the requirements of a real traffic. In particular, the constrains are the existence of a mixed transport network that contains one-way roads and the existence of a wider transport network, in which the only selected edges are used with possibility of effective passages. Data for the presented numerical experiments were both based on the real traffic values and artificially generated by the software Wolfram Mathematica. On the basis of these experiments performed in the computational environment XpressIVE was found, that this procedure can be appropriately applied in practice. As shown by the results, average savings computed by the mathematical model (5)-(9) are $13 \%$ in the case of variant $\mathrm{V} 2$ comparing to the variant V1. While using the real data, achieved savings of the total distance traveled are $4.3 \%$. Thanks to the effective passages, the objective function has been decreased from $280 \mathrm{~km}$ to $268 \mathrm{~km}$.

\section{Acknowledgements}

This paper was supported within Operational Programme Education for Competitiveness - Project No. CZ.1.07/2.3.00/20.0296 and programme for the support of applied research and experimental development, Technology Agency of the Czech Republic (EPSILON): Effective approaches to economical and adaptable systems of maintenance and operation of transport networks TH02010930. 


\section{References}

[1] BORCINOVA, Z., PESKO, S.: New Exact Iterative Method for the Capacitated Vehicle Routing Problem. Communications Scientific Letters of the University of Zilina, 18(3), 19-21, 2016.

[2] SIMCHI-LEVI, D., CHEN, X., BRAMEL, J.: The Logic of Logistics: Theory, Algorithms and Applications for Logistics Management. Springer, New York, 2014.

[3] SKIENA, S., PEMMARAJU, S.: Computational Discrete Mathematics. Cambridge University Press, New York, 2003.

[4] BONDY, A., MURTY, U.: Graph Theory. Springer, San Francisco, 2008.

[5] PELIKAN, J.: The Discrete Models in Operations Research. Professional Publishing, 2001.

[6] KOZEL, P., MICHALCOVA, S.: The Using of Linear Programming for Solving the Municipal Waste Collection Problem. Proceedings of 32nd International Conference Mathematical Methods in Economics, Czech Republic, 483-486, 2014.

[7] EISElT, H. A., GENDREAU, M., LAPORTE, G.: Arc Routing Problems, Part II: The Rural Postman Problem. Operational Research, 43(3), 399-414, 1995. https://doi.org/10.1287/opre.43.3.399

[8] KOZEL, P., MICHAlCOVA, S.: The Use of Linear Programming to Solve Routing Tasks in Practice. Proceedings of 33rd International Conference Mathematical Methods in Economics, Czech Republic, 395-400, 2015.

[9] GUERET, CH., PRINS, CH., SEVAUX, M., HEIPCKE, S.: Applications of Optimization with Xpress-MP. Blisworth: Dash Associates, United Kingdom, 2002.

[10] XPRESS-Mosel "User guide”. Blisworth: Dash Associates, United Kingdom, 2005.

[11] SOBEK, M.: Municipal Waste Collection Routes Optimization. Bachelor thesis (in Czech). VSB - TU Ostrava, Ostrava, 2018.

[12] SPACEK, F.: Mathematical Programming Application in Routing Problems Solving (in Czech). Master thesis. VSB - TU Ostrava, Ostrava, 2018.

[13] FRIEDRICH, V.: Mathematica for non-mathematician (in Czech). VSB - TU Ostrava, Ostrava, 2013.

[14] WOLFRAM, S.: The Mathematica Book. Cambridge University Press, New York, 1999. 